\title{
Estructura grupal,
}

interacción entre alumnos

y aprendizaje escolar

\section{César Coll *}

Universidad de Barcelona

RADICIONALMENTE, psicólogos y pedagogos han considerado la interacción profesor-alumno como la más decisiva para el logro de los objetivos educativos, tanto de los que se refieren al aprendizaje de contenidos como de los que conciernen el desarrollo cognitivo y social. De acuerdo con Johnson (198 ia), el énfasis casi exclusivo en la interacciọ́n profesor-alumno responde, al menos en parte, a la idea de que las relaciones que se establecen entre los alumnos en el transcurso de las actividades de aprendizaje tienen una influencia secundaria, cuando no indeseable o molesta, sobre el rendimiento escolar. Es obvia, por lo demás, la dependencia de esta idea de una concepción de la enseñanza que contempla al profesor como el agente educativo por excelencia encargado de transmitir el conocimiento y al alumno como un receptáculo más o menos activo de la acción transmisora del profesor. No es, pues, extraño que en este marco pedagógico se intente reducir a la minima expresión las relaciones alumno-alumno, sistemáticamente neutralizadas como fuente potencial de conductas perturbadoras en el aula, y que la planificación del aprendizaje repose sobre la primacía del trabajo individual de los alumnos y la interacción profesor-alumno.

Sin embargo, y sin que ello suponga en absoluto ignorar la importancia de la interacción profesor-alumno, suficientemente respaldada desde el punto de vista empírico, disponemos en la actualidad de pruebas suficientes que permiten afirmar sin vacilaciones que la interacción entre los alumnos no puede ni debe ser considerada un factor despreciable; por el contrario, todo parece indicar que juega un papel de primer orden en la consecución de las metas educativas. En efecto, durante las dos últimas décadas se han llevado a cabo numerosas investigaciones cuyos resultados coinciden en señalar (Johnson, i $98 \mathrm{r}$ b) que las relaciones entre alumnos - $o$ lo que es equivalente, la relación del alumno con sus compañeros, con sus iguales - inciden de forma decisiva sobre aspectos tales como el proceso de socialización en

* Dirección del autor: Dpto. de Psicología Evolutiva y Diferencial. Facultad de Psicología. Universidad de Barcelona. Avda. de Chile, s/n. 08028-Barcelona. 
general, la adquisición de competencias y de destrezas sociales, el control de los impulsos agresivos, el grado de adaptación a las normas establecidas, la superación del egocentrismo, la relativización progresiva del punto de vista propio, el nivel de aspiración e incluso el rendimiento escolar.

Pero el impacto de la interacción con el grupo de iguales sobre las variables mencionadas no es, por supuesto, constante ni en intensidad ni en sentido. Es decir, no basta con colocar los alumnos unos al lado de otros y permitirles que interactúen para obtener automáticamente unos efectos favorables. El elemento decisivo no es la cantidad de interacción, sino su naturaleza. La toma de conciencia de este hecho ha llevado a intensificar los esfuerzos dirigidos a identificar los tipos de organización social de las actividades de aprendizaje que posibilitan modalidades interactivas entre los alumnos especialmente favorables para la consecución de las metas educativas. De este modo, el paradigma dominante durante las últimos veinte anoos en el estudio de las relaciones alumno-alumno ha consistido en registrar y comparar las pautas interactivas y los resultados del aprendizaje que se obtienen mediante organizaciones grupales de las actividades escolares netamente contrastadas entre sí.

\section{La organización social de las actividades de aprendizaje}

Aunque esta tarea ha sido abordada desde diferentes perspectivas teóricas, las excelentes revisiones de Hayes (1976), Michaels (1977), Johnson y Johnson (1978), Slavin (1980) y Pepitone (1981), por citar sólo algunos, muestran que la atención de los investigadores se ha centrado prioritariamente en el estudio de tres formas básicas de organización social de las actividades escolares denominadas, respectivamente, cooperativa, competitiva e individualista.

En la perspectiva de la teoría del campo de Kurt Lewin, estas formas de organización han sido operativizadas atendiendo al tipo de interdependencia que existe entre los alumnos respecto a la tarea a realizar o el objetivo a conseguir en el transcurso de las actividades de aprendizaje. De este modo, siguiendo formulaciones previas de Lewin (1935) y de Deutsch (1949), Johnson (1981a) ha definido las tres organizaciones mencionadas como sigue. En una struación cooperativa los objetivos de los participantes están estrechamente vinculados, de tal manera que cada uno de ellos pueda alcanzar sus objetivos si y sólo si los otros alcanzan los suyos; los resultados que persigue cada miembro del grupo son, pues, beneficiosos para los restantes miembros con los que está interactuando cooperativamente. En una situación competitiva, por el contrario, los objetivos de los participantes están, también, relacionados, pero de forma excluyente: un participante puede alcanzar la meta que se ha propuesto sí y sólo si los otros no consiguen alcanzar los suyos; cada miembro del grupo persigue, pues, resultados que son personalmente beneficiosos, pero que son en principio perjudiciales para los otros miembros con los que está asociado competitivamente. Por último, en la situación individualista no existe relación alguna entre los objetivos que se proponen alcanzar los participantes: el hecho de que un participante alcance o no el objetivo fijado no influye sobre el hecho de que los otros participantes alcancen o no los 
suyos; se persiguen resultados individualmente beneficiosos, siendo irrelevantes los resultados obtenidos por los otros miembros del grupo.

La segunda perspectiva teórica desde la que se ha propuesto una definición operativa de la organización social de las tareas escolares es la del aprendizaje operante. Los autores que se sitúan en esta linea definen una organización como cooperativa cuando la recompensa que recibe cada participante es directamente proporcional a los resultados del trabajo del grupo. En una organización competitiva, por el contrario, un solo miembro del grupo recibe la recompensa máxima, mientras los otros reciben recompensas menores. Finalmente, en una organización individualista los participantes son recompensados en base a los resultados de su trabajo personal con total independencia de los resultados de los otros participantes. El criterio fundamental en este caso es, pues, la manera como se distribuyen las recompensas entre los participantes del grupo (reward structure) en lugar del tipo de interdependencia respecto a la consecución de los objetivos (goal structure).

Desde ambas perspectivas se han llevado a término numerosas investigaciones con el fin de estudiar la influencia de estos tres tipos de organización social de las actividades escolares sobre diferentes aspectos del proceso de enseñanza/aprendizaje, en particular de los que se refieren a la interacción que se establece entre los alumnos y a su relación con el nivel de rendimiento.

En cuanto al primer punto, los resultados son ampliamente concordantes. Las experiencias de aprendizaje cooperativo, comparadas con las de naturaleza competitiva e individualista, favorecen el establecimiento de relaciones entre los alumnos mucho más positivas que caracterizadas por la simpatía, la atención, la cortesía y el respeto mutuo, así como por sentimientos recíprocos de obligación y de ayuda. Estas actitudes positivas se extienden, además, a los profesores y al conjunto de la institución escolar. Contrariamente a lo que sucede en las situaciones competitivas, en las que los grupos se configuran sobre la base de una relativa homogeneidad del rendimiento académico de los participantes y suelen ser altamente coherentes y cerrados, en las situaciones cooperativas los grupos son, por lo general, más abiertos y fluidos y se constituyen sobre la base de variables como la motivación o los intereses de los alumnos.

En cuanto a la influencia de los tipos de organización social de las actividades de aprendizaje sobre el nivel de rendimiento de los participantes, los resultados son mucho menos claros. Tres puntos son particularmente polémicos. El primero consiste en determinar si las situaciones cooperativas dan lugar a un mejor rendimiento que las situaciones competitivas o a la inversa; el segundo, si las situaciones cooperativas dan lugar a un mejor rendimiento que las individualistas o a la inversa; y el tercero, si la competición intergrupos es un factor que incrementa o disminuye la efectividad de la cooperación intragrupos. Ante la situación un tanto confusa provocada por los resultados contradictorios de las investigaciones que han abordado estos puntos, Johnson, Maruyama, Johnson, Nelson y Skon (1981) han llevado a cabo una extensa y cuidadosa revisión, con el fin de pronunciarse sobre los mismos teniendo en cuenta las pruebas empíricas disponibles. Tanto por el tipo y volumen de las investigaciones revisadas como por la 
metodología empleada, este trabajo constituye un balance actualizado de la problemática y merece que nos detengamos brevemente en el mismo.

Dos son los objetivos principales de los autores: comparar los diferentes tipos de organización social de las actividades de aprendizaje en cuanto a su incidencia sobre el nivel de roml minto de los participantes; y aislar las variables supuestamente responn.ibles de esta incidencia desigual. Los resultados de 122 investigaciones, rcalizadas todas ellas con muestras de alumnos de Estados Unidos, son sometidos a un meta-análisis utilizando diversas técnicas. Como es sabido, el meta-análisis se aplica a un conjunto de investigaciones que estudian la misma problemática y que comparten las principales variables dependientes e independientes. El meta-análisis permite determinar la probabilidad de que los resultados de las diferentes investigaciones puedan atribuirse al azar, con lo que se obtiene una panorámica de conjunto de las tendencias surgidas por los resultados disponibles en un momento dado. Procediendo de este modo, Johnson y sus colegas llegan a unas conclusiones que pasamos a describir brevemente. Recordemos, una vez más, que, por las características del trabajo, estas conclusiones pueden ser consideradas un balance de los conocimientos actuales sobre el tema.

a) Las situaciones cooperativas son superiores a las competitivas en lo que concierne al rendimiento y a la productividad de los participantes. Esta relación se verifica cualquiera que sea el grupo de edad considerado (aunque es más fuerte en alumnos preuniversitarios) y la naturaleza del contenido (lenguaje, lectura, matemáticas, ciencias naturales, ciencias sociales, psicología, actividades artísticas, educación fisica). La superioridad se manifiesta también en tareas de formación de conceptos y de resolución de problemas. Sólo en el caso de tareas mecánicas las situaciones cooperativas no son superiores a las competitivas. La superioridad, en cambio, aumenta cuando la tarea a realizar consiste en la elaboración de un producto; también cuando hay una estimulación mutua entre los participantes, cuando hay un intercambio fluido de la comunicación y cuando tiene lugar una repetición verbal del material a aprender. Así pues, hay una serie de variables que afectan, que modulan la superioridad de la cooperación sobre la competición incrementándola o disminuyéndola según los casos. Sin embargo, cuando los autores llevan a cabo un análisis multivariado, las variables moduladoras mencionadas sólo permiten dar cuenta del 2 I por 100 de las varianza total observada en los resultados. Dicho de otro modo, la influencia de la cooperación sobre el nivel de rendimiento muestra una gran variabilidad que sólo en un porcentaje relativamente pequeño puede ser atribuido a la edad, al contenido, al tipo de tarea o a las otras variables identificadas. El hecho de que un 80 por 100 , aproximadamente, de la varianza total observada esté aún por explicar indica que la mayor parte de las variables que afectan la superioridad de la cooperación sobre la competición nos son todavía desconocidas.

b) La cooperación intragrupo con competición intergrupos es superior a la competición interpersonal en cuanto al rendimiento y a la productividad de los participantes. La superioridad es mayor cuando la tarea consiste en elaborar un producto y cuando el efectivo de los grupos es pequeño. Las variables moduladoras dan cuenta en este caso del 60 por 100 , aproximadamente, de la varianza total observada. 
c) Las situaciones cooperativas son superiores a las individualistas en cuanto al rendimiento y a la productividad. Esto es cierto para todos los grupos de edad y para todos los contenidos estudiados. La superioridad es mayor cuando la tarea a realizar no es de naturaleza mecánica, cuando se produce una relación tutorial entre los participantes y cuando la tarea no obliga a una división del trabajo. No obstante, estas variables moduladoras dan cuenta únicamente del 7 por Ioo de la varianza total observada.

d) La cooperación sin competición intergrupos es superior a la cooperación con competición intergrupo en cuanto al rendimiento y la productividad. Esta conclusión debe aceptarse con reservas, pues el número de investigaciones que han comparado directamente ambos tipos de organización social de las actividades escolares es muy reducido.

e) No se constatan diferencias significativas entre las situaciones competitivas $y$ las situaciones individualistas en cuanto al rendimiento y la productividad de los participantes. Los resultados son confusos y no es posible detectar una tendencia neta, pese a que algunos indicios sugieren una ligera superioridad de las situaciones competitivas cuando los esfuerzos individualistas se acompañan de una prohibición expresa de contacto y de interacción entre los participantes.

Resumiendo, la revisión de Johnson y sus colegas muestra que, en conjunto, la organización cooperativa de las actividades de aprendizaje, comparada con organizaciones de tipo competitivo e individualista es netamente superior en lo que concierne al nivel de rendimiento y de productividad de los participantes. Igualmente importante para comprender la dirección en la que se orientan los esfuerzos actuales en el estudio de las relaciones entre alumnos es el hecho de que las variables que afectan a la mayor o menor superioridad de la organización cooperativa de las tareas escolares siguen siendo desconocidas en gran parte.

\section{INTERACCION ENTRE IGUALES Y PROCESOS COGNITIVOS}

Este desconocimiento relativo no puede sorprender si se tienen en cuenta las siguientes consideraciones. En primer lugar, la mayoría de los trabajos empíricos que se han ocupado de las relaciones alumnoalumno se han centrado prioritariamente en el análisis de sus repercusiones sobre el proceso de socialización en general, o sobre algunos de sus componentes. El número de trabajos dirigidos a estudiar los efectos de la interacción entre iguales sobre los procesos cognitivos y los procesos de aprendizaje es mucho más reducido.

En segundo lugar, incluso cuando el análisis concierne a los efectos de los diferentes tipos de organización social de las actividades de aprendizaje sobre el rendimiento escolar, como sucede con las investigaciones revisadas por Johnson y sus colegas, la perspectiva, habitualmente adoptada, corresponde a lo que Doyle (1977) ha denominado "paradigma proceso-producto de la enseñanza», paradigma que se caracteriza por dejar de lado los factores motivacionales y cognitivos implicados en el aprendizaje. La adopción de este planteamiento puede explicar, al menos en parte, la imposibilidad de dar cuenta de la 
varianza total observada en el rendimiento de los alumnos bajo una misma forma de organización social de las actividades de aprendizaje. Una hipótesis plausible es que las variables moduladoras aún no identificadas pueden tener su origen precisamente en los procesos motivacionales y cognitivos de los alumnos.

En tercer.lugar, conviene tener presente que el estudio de las relaciones entre los alumnos y de su incidencia sobre el rendimiento escolar ha seguido tradicionalmente dos vías casi siempre disociadas. Por una parte, se investiga qué modalidades de interacción resultan favorecidas por tal o cual organización social de las actividades de aprendizaje; por otra, se investiga la posible repercusión del tipo de organización social adoptada sobre el nivel de rendimiento en la ejecución de la tarea; pero rara vez, casi nunca, se ponen en relación las modalidades interactivas observadas con el proceso de ejecución de la tarea.

Sin embargo, desde una óptica explicativa que aspire a superar el umbral de la mera constatación, parece básico comprender cómo se articulan las modalidades interactivas que se establecen entre los participantes con los procesos psicológicos subyacentes al aprendizaje y a la ejecución de las tareas escolares. Sólo así será posible valorar adecuadamente la importancia educativa de los diferentes tipos de organización social de las actividades escolares y, en último término, de la naturaleza de las relaciones que pueden establecer entre si los alumnos en el transcurso de las mismas.

En lo que sigue, vamos a ocuparnos de algunos intentos realizados en esta dirección, cuya finalidad última consiste, pues, en lograr una mejor comprensión de los mecanismos psicológicos, a través de los cuales las relaciones que se establecen entre los alumnos repercuten sobre los procesos de aprendizaje implicados en la relación de las tareas escolares.

\section{DE LA INTERACCION SOCIAL AL CONFLICTO SOCIOCOGNITIVO}

Un intento que ha demostrado ser particularmente fructifero y que encierra, a nuestro entender, grandes perspectivas de futuro es el que ha cristalizado en la hipótesis del conflicto sociocognitivo, que constituye en realidad una extensión y un replanteamiento de algunos aspectos de la teoría genética. Aunque Piaget ha formulado en repetidas ocasiones (1932, 1947) algunas hipótesis sobre el papel de la «co-operación» -es decir, la coordinación de operaciones- en el desarrollo intelectual y ha estimulado en sus publicaciones pedagógicas la adopción de métodos de enseñanza que favorezcan los intercambios entre los alumnos, ni él ni la mayor parte de sus seguidores han proseguido estas hipótesis ni las han sometido a verificación experimental. Asimismo, por razones que atañen a su manera de concebir el proceso de construcción del conocimiento, Piaget ha contribuido indirectamente a popularizar un tipo de análisis de la relación profesor-alumno que prevé que la autoridad del primero conducirá casi inevitablemente al segundo a adoptar mecánicamente sus explicaciones sin que medie construcción intelectual alguna. Esta visión limitada de la relación que 
puede darse entre un adulto y un niño, entre un profesor y un alumno, ha llevado a muchos psicólogos de la educación de orientación genética a fijarse de manera exclusiva y unilateral en las relaciones que el niño mantiene con su medio físico, en las relaciones que el alumno establece con los objetos, como si la construcción del conocimiento dependiera únicamente de estas relaciones.

Asi, en la mayoría de las aplicaciones pedagógicas de base piagetiana, el alumno es percibido como un ser socialmente aislado que debe descubrir por si solo las propiedades de los objetos e incluso de sus propias acciones, viéndose privado de toda ayuda o soporte que tenga su origen en otros seres humanos. La centración casi exclusiva en las interacciones entre el alumno y un medio esencialmente físico lleva aparejada un menosprecio por las interacciones del alumno con su medio social y, por supuesto, de los posibles efectos de estas últimas sobre la adquisición del conocimiento.

Sin embargo, desde mediados de los años setenta aproximadamente, un sector de la Escuela de Ginebra, encabezado por Doise, Muguy y, sobre todo, Anne Nelly Perret-Clermont ha cuestionado esta reducción y ha focalizado sus esfuerzos investigadores en el análisis de las relaciones que el niño mantiene con una parcela de su medio social, concretamente con sus compañeros e iguales. La idea de partida de estos autores es que, en el marco interpretativo de la teoría genética, el análisis de las relaciones entre iguales puede contribuir a enriquecer nuestra comprensión de los procesos que están en la base de la socialización y del desarrollo intelectual del ser humano.

\subsection{Conflicto cognitivo y desarrollo intelectual}

La mayoria de las investigaciones realizadas en esta perspectiva adoptan un mismo tipo de diseño (Perret-Clermont, 1981). En un primer momento se aplica a los sujetos de la muestra un pretest que consiste, invariablemente, en la resolución de una prueba operativa (conservación, inducción de leyes, coordinación de perspectivas, etc.) o de una tarea directamente analizable en términos de componentes operatorios. El análisis de las respuestas individuales en el pretest permite determinar, para cada sujeto, su nivel de dominio de las operaciones intelectuales implicadas en la resolución de la tarea. En una segunda sesión, que tiene lugar algunos días después, los sujetos del grupo experimental.son tomados en grupos de dos o tres para realizar, ahora colectivamente, una nueva tarea que implica el mismo tipo de dificultades cognitivas que la tarea del pretest. Finalmente, y tras un lapso de algunos días, tiene lugar una tercera sesión, de postest, en la que los sujetos abordan otra vez individualmente la misma tarea del pretest. De este modo, es posible examinar, mediante una comparación pretest-postest, los progresos logrados durante la actividad colectiva. Como es lógico, los sujetos del grupo control sólo realizan las sesiones de pretest y postest, o bien resuelven la tarea experimental individualmente.

Los resultados de estas investigaciones han sido expuestos con detalle por Perret-Clermont en un libro publicado en 1979 con el título La construcción de la inteligencia en la interacción social. Particularmente 
interesantes por su pertinencia para nuestra discusión son los tres puntos siguientes:

1. A menudo la ejecución colectiva de la tarea experimental da lugar a producciones más elaboradas, e incluso más correctas, que las que exhiben los mismos sujetos cuando trabajan individualmente. Esto se comprueba con tareas de diferente naturaleza: representación espacial (Doise, Mugny y Perret-Clermont, 1975), cambios de perspectiva (Doise y Mugny, 1978), coordinación de actividades motrices (Doise y Mugny, 1975), conservación de cantidades $y$ conservación del númeri (Perret-Clermont, 1979), etc. En ocasiones, la superioridad de la producción grupal se debe a que uno de los miembros impone a los restantes una solución más correcta, pero, con frecuencia, esto no es asi: como afirma Doise, lo que sucede es que el simple hecho de actuar conjuntamente, cooperativamente, obliga a todos los miembros del grupo a estructurar mejor sus actividades, a explicitarlas, a coordinarlas, sin que la responsabilidad pueda atribuirse en exclusiva a uno de los participantes.

2. ${ }^{\circ}$ Es interesante destacar que el trabajo colectivo no siempre da sus frutos de manera inmediata durante la realización en grupo de la tarea, sino que en algunos casos se manifiestan en las producciones individuales del postest. Cuando esto sucede, la interacción social que se produce durante la realización colectiva de la tarea, parece ser, siempre en opinión de los autores de estos trabajos, el punto de partida de una coordinación cognitiva cuyos efectos se manifiestan con posterioridad en las producciones individuales.

$3 .^{\circ}$ Hay dos situaciones-tipo en las que no se observa progreso alguno en las competencias intelectuales de los participantes en la actividad grupal: cuando uno de los miembros impone su punto de vista a los otros, que se limitan a adoptarlo; y cuando todos los miembros tienen el mismo punto de vista sobre la realización de la tarea. Por el contrario, hay casi siempre un progreso cuando en el transcurso de la realización grupal de la tarea se produce una confrontación de puntos de vista moderadamente divergentes sobre la manera de abordarla. Importa poco que la confrontación se produzca entre puntos de vista correctos e incorrectos, o bien que los puntos de vista sean incorrectos por ambos lados. Dicho de otro modo, los resultados sugieren que el factor determinante para que se produzca un progreso intelectual es la posibilidad de confrontar los puntos de vista propios con otros ajenos, independientemente del grado de corrección de ambos, que es hasta cierto punto un aspecto secundario, al menos en lo que concierne a la interacción entre iguales.

Este breve resumen de algunos de los resultados obtenidos por Perret-Clermont y sus colegas basta para comprender la naturaleza y el alcance de la hipótesis que formulan respecto a los mecanismos responsables de los progresos intelectuales observados como consecuencia de la interacción grupal o, más concretamente, de la interacción entre iguales. La idea esencial es la necesidad de una confrontación entre puntos de vista moderadamente divergentes; la existencia de centraciones diferentes, a propósito de una misma situación o tarea, se traduce, gracias a la exigencia de una actividad grupal común, en un conflicto sociocognitivo que moviliza y fuerza las reestructuraciones intelectuales y, con ello, el progreso intelectual. Para que los niños puedan 
elaborar conjuntamente una noción o resolver un problema no es necesario que uno de los participantes la domine ya o que conozca la solución. Basta con que aborden estas tareas con puntos de vista divergentes $y$ con que tengan las competencias intelectuales mínimas que exige la estructura de la noción o del problema.

El concepto de conflicto cognitivo no es, por, supuesto, nuevo en lá teoría genética. En efecto, aparece ya en las primeras publicaciones de Piaget en los años veinte, implícito en el proceso de equilibración mediante el cual este autor explica el carácter teleonómico del desarrollo. Asimismo, y más recientemente, Inhelder, Sinclair y Bovet (1974) lo han reformulado y profundizado con el fin de explicar el aprendizaje de las estructuras operatorias. Pero, en lo que podriamos llamar la versión ortodoxa piagetiana, el conflicto cognitivo aparece básicamente como resultado de la falta de acuerdo entre los esquemas de asimilación del sujeto y la constatación de los observables físicos correspondientes, o bien como resultado de las contradicciones internas entre los diferentes esquemas del sujeto (Piaget, 1975). En el caso de Perret-Clermont y sus colegas, la naturaleza del conflicto es sustancialmente distinta, pues se concibe como el resultado de la confrontación entre esquemas de sujetos diferentes que se produce en el transcurso de la interacción social. De aquí la denominación de conflicto sociocognitivo.

\subsection{Conflicto cognitivo y construcción del conocimiento}

Todas las investigaciones de Doise, Mughy y Perret-Clermont a las que nos hemos referido hasta el momento presentan la particularidad de utilizar tareas directamente analizables en términos de componentes operatorios. Ahora bien, ¿puede generalizarse la hipótesis del conflicto sociocognitivo a la adquisición de contenidos escolares, es decir, a la adquisición de conocimientos que no son reductibles en su totalidad a componentes operatorios, sino que poseen una fuerte carga social y cultural?

Esta pregunta está en la base de las preocupaciones actuales de algunos psicopedagogos ginebrinos como Schubauer-Leoni, Jean Brun y la misma Perret-Clermont. Aunque no se dispone todavía de resultados concluyentes, si que existen ya algunas indicaciones de interés. Veamos brevemente la manera de plantear el problema $y$ algunos resultados a los que llegan Schubauer-Ieoni y Perret-Clermont (1980) en una de las investigaciones realizadas en esta perspectiva.

El objetivo de este trabajo era estudiar el papel del contexto interpersonal, y más concretamente de la interacción y de la comunicación social, en el aprendizaje de un campo de contenidos escolares muy particular: el de la actualización por el alumno de un código matemático como la escritura de ecuaciones del tipo $a+b-c=x$. Es sabido que los alumnos de siete-ocho años a los que se ha enseñado las operaciones de adición y sustracción, así como su escritura en forma de ecuaciones, pese a resolver correctamente los ejercicios clásicos de control, tienen dificultades para utilizar espontáneamente la notación ecuacional cuando deben representar por escrito un conjunto de manipulaciones efectuadas en su presencia. Por ejemplo: se cogen dos caramelos y se meten en un recipiente; se repite la acción con cuatro caramelos; 
Einalmente, se cuentan los caramelos que hay en el recipiente (seis). Pues bien, los niños de siete-ocho años suelen representar esta serie de manipulaciones de forma gráfica o verbal, pero casi nunca urilizando la notación ecuacional que seria, en este caso, $2+4=6$.

En la experiencia de Schubauer-Leoni y Perret-Clermont, iz alumnos de ocho años que no utilizan espontáneamente la notación ecuacional, pese a conocerla y a resolver correctamente los ejercicios habituales -para determinar lo cual, lógicamente, se les ha sometido a una exploración previa-, son repartidos en cuatro grupos experimentales: en el primero $\left(G E_{1}\right)$, los sujetos, en grupos de dos, deben codificar conjuntamente por escrito una manipulación como la del ejemplo, con el fin de comunicarla a otro niño del mismo nivel, al cual se le pide que la interprete en presencia de los autores de la codificación y del experimentador (en el GE se da, pues, interacción - codificación conjunta- y comunicación-presencia de un compañero que debe interpretar la codificación efectuada); en el grupo experimental dos $\left(G_{2}\right)$, los sujetos codifican la manipulación efectuada en su presencia, pero el único destinatario es el experimentador, que no emite ningún juicio al respecto (hay, pues, interacción, pero no comunicación a un compañero); en el $\mathrm{GE}_{3}$, la codificación es individual y se hace con el fin de comunicarla a un compañero que, como en el caso del $\mathrm{GE}_{1}$, la interpreta en presencia de su autor y del experimentador (no hay, pues, interacción, pero sí comunicación); por último, en el $\mathrm{GE}_{4}$ los sujetos hacen la codificación individualmente y sólo es vista por el experimentador que se abstiene de comentarla. El diseño se complementa con un pretest y un postest, ambos con dos pruebas: una prucba operatoria de composición aditiva del número y una prueba de codificación similar a la de la tarea experimental.

Grosso modo, los resultados muestran un efecto general de aprendizaje en los cuatro grupos: 82 por roo de los sujetos dan una formulación más explícita y detallada de las manipulaciones en el postest que en el pretest. Sin embargo, se abservan diferencias significativas entre los cuatro grupos. El grupo que muestra mayores progresos es el que realiza la tarea en una situación de interacción con comunicación a un tercero; le sigue el grupo en el que hay interacción sin comunicación; con muy poca diferencia, el grupo en el que ha habido una codificación individual con comunicación a un compañero; finalmente, el grupo que muestra menos progresos es aquel en el que los sujetos han realizado una codificación individual sin comunicación a un compañero. Las diferencias son significativas en todos los casos excepto entre $\mathrm{GE}_{2}$ y $\mathrm{GE}_{3}$.

Estos resultados sugieren que diferentes tipos de interacción social incitan a una utilización más o menos frecuente y más o menos correcta de un formalismo matemático aprendido. La interacción entre iguales y la comunicación a un tercero, tal como son activadas en el $G_{1}$, parecen jugar un papel importante en la utilización de la escritura ecuacional anteriormente aprendida. Schubauer-Leoni y Perret-Clermont interpretan estos resultados con la ayuda de la hipótesis del conflicto sociocognitivo. No obstante, si bien esta hipótesis permite dar vueltas del progreso experimentado por los grupos en los que se da una interacción, es decir, donde hay una codificación conjunta de las manipulaciones, su pertinencia para explicar el efecto de la comunica- 
ción a un compañero es mucho menos obvia. ¿Por qué el hecho de elaborar la codificación pensando que debe ser entendida por otra persona da lugar a producciones más explícitas, más detalladas y más correctas? Los autores evitan plantearse esta pregunta que, sin embargo, encuentra una respuesta plausible en el marco de la hipótesis del paso de la regulación interpsicológica a la regulación intrapsicológica a la que nos referiremos más adelante.

\subsection{Conflicto cognitivo y controversias conceptuales}

Digamos aún que la hipótesis del conflicto.sociocognitivo es compatible con los resultados de otras investigaciones realizadas al margen de los supuestos teóricos de la psicología genética e interpretados por sus autores desde otras perspectivas (cf. el articulo de Webb incluido en este dossier). Particularmente interesante a este respecto es la formulación, muy próxima a la del conflicto sociocognitivo, a la que han llegado Johnson y sus colaboradores a partir de una serie de investigaciones sobre el efecto de las controversias que se producen en el transcurso de la interacción entre iguales durante la realización de tareas escolares.

Se dice que existe una controversia cuando se produce una incompatibilidad entre las ideas, informaciones, opiniones, creencias, conclusiones o teorias de los miembros de un grupo y hay, además, una voluntad de llegar a un acuerdo, a una postura común. Nótese que la diferencia entre conflicto y controversia reside precisamente en la voluntad de superar las discrepancias que están en la base del conflicto. Desde el punto de vista psicopedagógico, la cuestión clave consiste en transformar los conflictos - inevitables cuando se permite una interacción fluida entre los alumnos- en controversias; o más exactamente, en controversias que puedan ser resueltas de forma constructiva. En efecto, se ha demostrado que las controversias pueden tener efectos positivos sobre la socialización, el desarrollo intelectual y el rendimiento escolar, pero pueden tener también efectos negativos si no se manejan y se resuelven adecuadamente. Algunas condiciones para que las controversias entre alumnos sean potencialmente constructivas han sido establecidas mediante estudios empíricos. Asi, por ejemplo, Johnson ( $198 \mathrm{rb}$ ) cita las siguientes:

- Cuanto más heterogéneos (en cuanto a personalidad, sexo, aptitudes, conocimientos previos, estrategias de razonamiento, etcétera) son los participantes, mayor es la probabilidad de que surjan conflictos y controversias.

- Cuanto más relevante es la información disponible y más motivados y capaces intelectualmente son los alumnos, mayor es la probabilidad de que las controversias tengan efectos constructivos.

- Cuanto mayor es la tendencia de los alumnos a discrepar sin atribuir el origen de la discrepancia a la incompetencia o a la falta de información de los oponentes, más constructivos son los efectos de la controversia.

- Cuanto más elevada es la perspectiva teórica y cuanto mayor es el volumen y la calidad de los conocimientos de los oponentes, más constructivos son los efectos de la controversia. 
- Cuanto más capaces son los oponentes de relativizar su propio punto de vista - es decir, de adoptar la perspectiva de los demás-, mayor es la probabilidad de que la controversia se resuelva constructivamente.

- Cuanto más cooperativa es la situación en que tiene lugar la controversia, mayores son sus efectos constructivos. Todo parece indicar que las organizaciones sociales de tipo cooperativo favorecen la comunicación entre los oponentes, contribuyen a establecer un clima de aceptación y conducen a la búsqueda de puntos de vista de contacto entre posturas con,trapuestas.

Según Johnson y Johnson (1979), en la controversia resuelta de forma constructiva se produce un conflicto conceptual que, a su vez, genera sentimientos de incertidumbre y un desequilibrio cognitivo y afectivo en los participantes; este desequilibrio lleva a buscar nuevas informaciones y a analizar desde perspectivas novedosas las informaciones disponibles. Salvando las diferencias terminológicas, es obvio el paralelismo de esta formulación con la que proporciona la hipótesis del conflicto sociocognitivo.

En resumen, esta hipótesis cuenta en la actualidad con una base empírica de considerable solidez, enriquecida, como hemos apuntado, por la convergencia con otras interpretaciones elaboradas al margen de las coordenadas teóricas de la psicología genética. Sin embargo, conviene también llamar la atención sobre algunas limitaciones de la misma igualmente importantes. Ya hemos aludido a su dudosa pertinencia para explicar una parte de los resultados obtenidos por los propios Schubauer-Leoni y Perret-Clermont (1980), lo que sugiere que la confrontación de puntos de vista divergentes, si bien es uno de los caminos a través de los cuales la interacción entre los alumnos repercute sobre el desarrollo intelectual y el aprendizaje escolar, no es probablemente el único camino.

Pero hay otra limitación todavía más esencial: los obstáculos que encuentra la hipótesis del conflicto sociocognitivo para detallar pormenorizadamente los mecanismos psicológicos responsables de la influencia que ejerce la interacción y la comunicación entre iguales sobre la adquisición y la utilización del conocimiento. Para superar estos obstáculos parece necesario no sólo ampliar el marco teórico de referencia, sino también dotarse de los instrumentos adecuados que permiten observar detalladamente el proceso de coordinación social que tiene lugar entre los participantes durante la realización colectiva de la tarea; sólo así se puede aspirar a discernir las variables interactivas directamente responsables del progreso cognitivo. En esta doble dirección se orientan los esfuerzos actuales entre los que cabe destacar los trabajos inspirados en las tesis de Vygotski y en otras aportaciones de la psicología soviética (Forman, 1981; 1982; Forman y Cazden, en prensa, etc.) que, pese a encontrarse todavía en una fase incipiente, han protagonizado ya aportaciones sustanciales a la problemática que nos ocupa. 
La inclusión en este número del excelente trabajo de Forman y Cazden, aconseja una máxima brevedad en esta parte de la exposición. Sin embargo, creemos que el interés de este nuevo planteamiento y las enormes perspectivas de futuro que encierra para el estudio de las relaciones entre los alumnos, justifican, aún a riesgo de caer en repeticiones inevitables, algunos comentarios de indole general. Tres son los puntos que queremos destacar: el cambio metodológico, las ideas del marco teórico de referencia y la demostración de que existen otros mecanismos mediadores -además del conflicto sociocognitivoentre la interacción alumno-alumno y el aprendizaje escolar.

Empezando por las cuestiones metodológicas, decíamos ya en la introducción que el paradigma dominante durante los últimos veinte años en el estudio de las relaciones entre alumnos ha consistido en registrar y comparar las pautas interactivas y/o los resultados del aprendizaje que se obtienen a partir de organizaciones grupales de las actividades escolares netamente contrastadas entre sí; y añadiremos más adelante que dicho estudio ha seguido dos vías casi siempre disociadas: o bien se analiza la repercusión de la organización social adoptada sobre las modalidades de interacción que se establecen entre los alumnos, o bien se analiza su repercusión sobre el nivel de rendimiento en la ejecución de la tarea, pero casi nunca se tiene en cuenta la articulación de ambos aspectos.

Esta disposición es patente en las investigaciones que comparan las estructuras cooperativa, competitiva e individualista, pero, como señalan con acierto Forman y Cazden, se encuentran también en las investigaciones de Perret-Clermont y sus colaboradores. En el trabajo comentado de Schubauer-Leoni y Perret-Clermont (1980), por ejemplo, los sujetos que codifican las manipulaciones en unas condiciones de "interacción social» - en grupos de dos- son tratados de forma homogénea en el análisis de los resultados, dando por supuesto que las pautas interactivas que se establecen entre los dos participantes o bien son idénticas en todas las parejas, o bien no repercuten sobre la codificación elaborada en común. Este supuesto es el que justifica que se comparen las mejoras logradas por los diferentes grupos experimentales entre el pretest y el postest. Sin embargo, nada autoriza a pensar que dichas mejoras se deban únicamente a la presencia o ausencia de la variable independiente "interacción social»; es posible, e incluso probable, que la interacción social se haya concretado en las diversas parejas en pautas interactivas de diferente naturaleza, con repercusiones directas sobre el progreso manifestado por los sujetos en el postest. Sería ingenuo, además, suponer que estas pautas interactivas permanecen constantes para una pareja determinada durante toda la sesión experimental; mucho más razonable parece pensar que el tipo de interacción que se establece entre los partieipantes va evolucionando a medida que se avanza en la resolución de la tarea.

Comprender los mecanismos psicológicos que median entre la interacción alumno-alumno y los procesos cognitivos implicados en las tareas de aprendizaje escolar exige como mínimo tres requisitos: observar cómo evolucionan las pautas interactivas que se establecen 
entre los participantes, observar cómo evoluciona el proceso de realización de la tarea y, finalmente, observar cómo se coordinan y se condicionan mutuamente ambos aspectos. Esto se consigue efectuando registros observacionales de un gran nivel de precisión que son sometidos posteriormente a un análisis microgenético. Muy brevemente, el análisis microgenético (Wertsch y Stone, 1978; Zinchenko, 1981; etcétera) consiste en analizar con detalle la constitución y las modificaciones sucesivas de una destreza, concepto o estrategia en el transcurso de una unidad temporal más o menos amplia. El punto de partida de este tipo de análisis reside en identificar las unidades funcionales, los componentes básicos, de la destreza, concepto o estrategia que son objeto de estudio y en examinar cómo estas unidades interactúan y evolucionan durante el período temporal prefijado. En el trabajo de Forman y Cazden se encuentran unas bellas ilustracciones de aplicación del análisis microgenético.

En cuanto al marco teórico de referencia, la novedad reside en la estrecha conexión que se postula entre el desarrollo intelectual y cognitivo por una parte, y la interacción social por la otra. Aunque a primera vista este postulado está también prcsente en la hipótesis del conflicto sociocognitivo, la naturaleza de la explicación es sustancialmente distinta en ambos casos. En la formulación de Perret-Clermont y sus colegas, tributaria de la teoría general de Jean Piaget, la interacción social favorece el desarrollo del razonamiento lógico y la adquisición de contenidos escolares, gracias a un proceso de reorganización cognitiva provocado por el surgimiento de conflictos y por su superación; en la formulación de Forman y Cazden, inspirada en las ideas de Vygotski y en otras aportaciones de la psicología soviética, la interacción social es el origen y el motor del aprendizaje y del desarrollo intelectual gracias al proceso de interiorización que hace posible.

Como es sabido, Vygotski propuso el concepto de zona de desarrollo próximo para explicar el desfase existente entre la resolución individual y social de problemas y tareas cognitivas: a menudo, las personas somos capaces de resolver problemas o de efectuar aprendizajes nuevos cuando contamos con la ayuda de nuestros semejantes, pero, en cambio, no conseguimos abordar con éxito estas mismas tareas cuando disponemos únicamente de nuestros propios medios. La zona de desarrollo próximo es «la diferencia entre el nivel de las tareas realizables con la ayuda de los adultos y el nivel de las tareas que pueden realizarse con una actividad independiente" (Vygotski, 1973, pág. 34.) El aprendizaje se sitúa precisamente en esta zona: lo que en un principio el niño es capaz de hacer o conocer únicamente con la ayuda del adulto, con el aprendizaje llega a ser capaz de hacerlo o conocerlo por si solo. Se llega de este modo a concebir las relaciones entre desarrollo, aprendizaje y enseñanza en una perspectiva radicalmente distinta a la de Piaget y sus colaboradores:

«El proceso de desarrollo no coincide con el de aprendizaje, el proceso de desarrollo sigue al de aprendizaje, que crea el área de desarrollo potencial” (Vygotski, 1973, pág. 39).

«El rasgo esencial del aprendizaje es que engendra el área de desarrollo potencial, o sea, que hace nacer, estimula y activa en el niño un grupo de procesos internos de desarrollo dentro del marco de las interrelaciones con otros, que a continuación son absorbidos por el curso interno del desarrollo y se convierten en adquisiciones 
internas del niño (...). El proceso de aprendizaje es una fuente de desarrollo que activa nuevos procesos que no podrian desarrollarse por si mismos sin el aprendizaje" (Ibidem, pág. 37).

"Una enseñanza orientada hacia una etapa de desarrollo ya realizado es ineficaz desde el punto de vista del desarrollo general de! niño, no es capaz de dirigir el curso del desarrollo, sino que le va a la zaga (...). La única buena enseñanza es la que se adelanta al desarrollow (Ibidem, págs. 35-36).

En la interacción social, el niño aprende a regular sus procesos cognitivos siguiendo las indicaciones y directrices de los adultos, produciéndose un proceso de interiorización mediante el cual lo que puede hacer o conocer en un principio con la ayuda de ellos (regulación interpsicológica), se transforma progresivamente en algo que puede hacer o conocer por sí mismo (regulación intrapsicológica). El origen social de la cognición, el estrecho vínculo existente entre la interacción social por un lado y el aprendizaje y desarrollo por el otro, queda totalmente de manifiesto en lo que Vygotski considera la ley más importante del desarrollo del psiquismo humano:

"Todas las funciones psicointelectivas superiores aparecen dos veces en el curso del desarrollo del niño: la primera ve\% en las actividades colectivas, en las actividades sociales, o sea, como funciones interpsiquicas; la segunda, en las actividades individuales, como propiedades internas del pensamiento del niño, o sea, como funciones intrapsiquicas" (Ibidem, pág. 36).

No podemos dejar de mencionar, en estas breves pinceladas del trasfondo teórico de la hipótesis de la regulación, el papel decisivo que juega el lenguaje. El lenguaje es el instrumento regulador por excelencia de la acción y del pensamiento, pero, además, el lenguaje adquiere esta función reguladora cuando es utilizado en toda su potencialidad instrumental -es decir, «cuando es utilizado como instrumento para llevar a cabo diferentes formas de comportamiento" (Levina, i 98 I, pág. 290) - en el marco de la interacción social. Junto a la función comunicativa, el lenguaje tiene también una función reguladora de los procesos cognitivos, pues, parafraseando a Levina, el intento de formular verbalmente la representación propia con el fin de comunicarla a los demás obliga a reconsiderar y reanalizar lo que se pretende transmitir. El proceso de interiorización marca el paso de la regulación externa, social, interpsicológica, de los procesos cognitivos mediante el lenguaje de los demás, a la regulación interiorizada, individual, intrapsicológica, de los procesos cognitivos mediante el lenguaje interno.

Volviendo ahora al tema principal de este apartado, el trabajo de Forman y Cazden es un intento de generalizar los plantẹamientos anteriores, formulados en un principio en el contexto de la interacción adulto-niño, a la interacción entre iguales. También en este caso el lenguaje es considerado como la piedra de toque del proceso de interiorización, respondiendo a las mismas motivaciones (necesidad de comunicar a los compañeros la representación propia) y dando lugar a las mismas consecuencias (necesidad de revisar la representación y el punto de vista propios). En la interacción entre iguales encontramos todos los peldaños intermedios entre el hecho de dirigir la acción y los procesos mentales propios a partir del lenguaje de los demás (regulación interpsicológica) y el hecho de dirigir la acción y los procesos 
mentales propios a partir del lenguaje interno (regulación intrapsicológica). Más aún, la interacción entre iguales, debido a la simetría de principio en las relaciones entre los participantes, ofrece unas condiciones óptimas para que los alumnos aprendan a utilizar el lenguaje en todo su valor instrumental; es decir, a utilizar el lenguaje de los compañeros para guiar las acciones propias, a utilizar el lenguaje propio para guiar las acciones de los compañeros y, sobre todo, a utilizar el lenguaje interior para guiar las propias acciones.

La hipótesis de la función reguladora del lenguaje y del establecimiento progresivo de mecanismos de regulación intrapsicológica a partir de las regulaciones interpsicológicas que se producen en la interacción entre iguales permite dar cuenta de algunos resultados empíricos difícilmente interpretables en el marco de la hipótesis del conflicto sociocognitivo. Es el caso, por ejemplo, de los progresos observados en la investigación de Schubauer-Leoni y Perret-Clermont cuando los sujetos deben elaborar una codificación de las manipulaciones efectuadas en su presencia con el fin de comunicarla a otro compañero del mismo nivel. Es el caso también de los resultados expuestos recientemente por Webb (1983), según los cuales el hecho de proporcionar y de recibir explicaciones de los compañeros en el transcurso de la interacción tiene efectos cognitivos favorables para el emisor y el receptor, respectivamente; efectos que desaparecen cuando lo que se transmite o se recibe son soluciones ya hechas a la tarea planteada. $O$ aún de los efectos cognitivos favorables que, en las relaciones tutoriales - situaciones en las que un alumno enseña o instruye a sus iguales-, se derivan para el niño-tutor del hecho de formular instrucciones verbales o explicaciones dirigidas a sus compañeros. $O$, finalmente, de las repercusiones positivas sobre el proceso de resolución de problemas que tiene el establecimiento de relaciones cooperativas entre los miembros del grupo, relaciones que se caracterizan por la coordinación de los roles asumidos por los participantes, el control mututo del trabajo y el reparto de responsabilidades.

En todas estas situaciones es más bien raró observar conflictos abiertos entre puntos de vista divergentes, por lo que los efectos que tienen las modalidades interactivas en juego sobre los procesos cognitivos implicados en la realización de las tareas no se dejan explicar fácilmente mediante la hipótesis del conflicto sociocognitivo. Más adecuado parece interpretar estos resultados mediante la hipótesis de la regulación, es decir, apelando a la exigencia de revisar y reanalizar el punto de vista propio impuesta por las verbalizaciones que sirven de instrumento a la interacción.

\section{ร. COMENTARIOS FINALES}

Aunque difícilmente puede concluirse a propósito de una problemática como la que nos ocupa, cuyo estudio es todavia incipiente y fragmentario y que está en plena efervescencia de aportaciones, nuestras últimas frases estarán dedicadas a intentar un balance sintético del camino recorrido y a enunciar, no menos sintéticamente, algunas perspectivas respecto al camino que queda por recorrer. 
podemos albergar duda alguna sobre la importancia de las relaciones entre los alumnos para el logro de los objetivos educativos, tanto de los estrictamente socioafectivos como de los instrumentales y de contenido. Las investigaciones realizadas permiten afirmar que, en general, la organización cooperativa de las actividades escolares tiene unos efectos más favorables sobre el aprendizaje que la organización competitiva $o$ individualista.

Asimismo, se han podido identificar tres tipos de relaciones entre los alumnos que, bajo determinadas condiciones, pueden tener una influencia particularmente positiva sobre el nivel de rendimiento y los resultados del aprendizaje: cuando existen puntos de vista moderadamente divergentes sobre la tarea a realizar y se produce un conflicto entre los mismos; cuando uno de los participantes enseña o instruye a los otros proporcionándoles explicaciones, instrucciones o directrices sobre la tarea, y cuando hay una coordinación de los roles asumidos por los miembros del grupo, un control mutuo del trabajo y un reparto de responsabilidades de la ejecución de la tarea.

Desáe el punto de vista de ía interpretación de los mecanismos a través de los cuales se produce esta influencia, dos explicaciones distintas comparten la escena. De acuerdo con la primera, los conflictos sociocognitivos (en la terminología de Perret-Clermout y sus colegas) o las controversias (en la terminologia de Johnson y Johnson), que se producen como resultado de la confrontación de puntos de vista, estimulan los procesos cognitivos y fuerzan la búsqueda de nuevas informaciones o la reconsideración de las que ya se poseen. De acuerdo con la segunda, la ayuda y el soporte mutuos que proporciona la situación interactiva fuerza la reestructuración intelectual; primero, mediante la regulación reciproca que ejercen entre sí los participantes; después, y de forma progresiva, mediante la autorregulación individual.

Respecto a esta doble explicación cabe hacer al menos tres observaciones. La primera es que la hipótesis del conflicto sociocognitivo y la hipótesis del paso gradual de una regulación interpsicológica a una regulación intrapsicológica tienen numerosos puntos en común, sobre todo en lo que concierne a los supuestos básicos relativos a la naturaleza del funcionamiento cognitivo. La segunda es que, pese a ello, las dos explicaciones no son reductibles entre sí; la hipótesis del conflicto no permite dar cuenta de los efectos positivos que se constatan en las situaciones tutoriales o en las situaciones de trabajo grupal genuinamente cooperativo; inversamente, la hipótesis de la regulación se aplica con incomodidad a los efectos favorables de la superación de conflictos y controversias conceptuales. En consecuencia, y es la tercera observación, la postura más razonable en la fase actual de nuestros conocimièntos consiste, a nuestro juicio, en conjeturar que ambas hipótesis se aplican a dos mecanismos distintos, lo que no quiere decir opuestos o excluyentes. En este sentido, no hay ninguna razón que autorice a pensar que los tres tipos de relaciones identificadas sean los únicos susceptibles de incidir favorablemente sobre el aprendizaje escolar. No es arriesgado suponer que las investigaciones futuras mostrarán tal vez que esta incidencia puede producirse también con otros tipos de relaciones entre alumnos. Cuando esto suceda, es posible que ninguna de las dos hipótesis resulte adecuada para explicar los nuevos hallazgos; pero, por el momento, son sin 
ninguna duda las que mejor permiten dar cuenta de los resultados disponibles.

Finalmente, desde el punto de vista de las perspectivas de futuro, el camino que se vislumbra es el de un análisis aun más fino y detallado de la articulación entre procesos interactivos y conductas de ejecución de la tarea. Este propósito tiene implicaciones metodológicas importantes no exentas de dificultades que deberán afrontarse y resolverse. El análisis microgenético parece a priori un instrumento más adecuado para lograr este objetivo que otros métodos de análisis más tradicionales, o no tan tradicionales. El análisis microgenético es suceptible de proporcionar informaciones interesantes sobre una multitud de cuestiones aún no resueltas. Por ejemplo, sobre la importancia de la dimensión temporal (los distintos tipos de interacción no tienen probablemente los mismos efectos en las sucesivas fases de ejecución de la tarea); sobre la identificación de los factores responsables de que los tres tipos de relaciones mencionados no tengan en ocasiones repercusiones favorables sobre el aprendizaje, o que tengan repercusiones favorables de diversa intensidad; o aun sobre la identificación de otras variables moduladoras de la interacción alumno-alumno no estrictamente cognitivas, como la motivación, el nivel de ansiedad de los participantes, sus expectativas de éxito o su autoconcepto. Sin embargo, para que estas posibilidades que encierran las técnicas de análisis microgenético lleguen a concretarse, será necesario que alcancen un mayor grado de desarrollo y de perfección del que tienen en el momento actual.

Cabe esperar, no obstante, que el trabajo cooperativo, el intercambio de informaciones y la confrontación de puntos de vista divergentes permitirá a los psicólogos de la educación superar estas dificultades y progresar, en un futuro próximo, hacia una mejor comprensión de los mecanismos a través de los cuales la interacción entre los alumnos repercute sobre el aprendizaje escolar.

\section{Resumen}

La influencia sobre el proceso de socializacion de las relaciones que se establecen entre los alumnos, asi como su importancia para el logro de los objetivos socio-afectivos, es un becho reconocido desde bace tiempo. En cambio, los ejectos de estas relaciones solire el aprendizaje escolar es un aspecto mucho mas discutido, tal rez como consecuencia del modelo pedagogico imperante que contempla al profesor como el unico agente educativo. In el articulo se rerisan algunos arances conseguidos durante los tiltimos años en ef estudio de la interaccion alumno-alumno $\mathrm{j}$ de su influsencia sobre el nivel de rendimienlo $\mathrm{y}$ los resultados del aprendizaje. Se presta especial atención a los resultados de las iniestigaciones que ocuparon las diferentes formas de organización social de las actividades escolares -cooperacion, competición e individualismo-, asi como a algunos tipos de modalidades interactitas --relación tutorial, confrontación de puntos de rista divergentes. controtersias conceptuales y trabajo grupai cooperativo-, que pueden llegar a tener unos efectos particularmente benéficos para el aprendizajt. Isstos resultados son examinados a la luz de unos marcos explicativos diferentes, la hipotesis del conflicto sociocognitivo, la bipótesis de la regulación, para terminar enunciando algunas perspectivas de futuro solore las numerosas cuestiones pendientes de respuesta.

\section{Summary}

The influence of pupils social relations on their own process of socialization and its relevance in the accomplishment of socio-affective purposes is a well known matter. But the effects of these relations on school learning is a rather controversial matter, attribuable perbaps to the prevalent educational system, that considers the teacher as the unique educational agent. In this paper, some recent advances in the pupil-pupil interaction and its influence on school c -hievement and learning are considered. Special attention is devoted to research outputs about comparisons between differents forms of social organization of the school activities - cooperation, competition, individualism - and about some kinds of 
improvement on school learning. These results are examined from two different models. On one band, the social cognitive conflict view, and on the other band the regulation bypotbesis. Finally, some future perspectives without a present answer are examined.

\section{Résumé}

Depuis longtemps on connait I'influence sur le processus de socialisation qui s'etablissent entre les éleves, ainsi que leur importance pour atteindre les objectifs socio-affectifs de l'enseignement. En contrepartie, les effets de ces relations sur lapprentissage scolaire sont encore discutes; la nature du modele pédagogique dominant, qui voit le professeur comme le seul agent educatif. est peut-être une des raisons de cet état des choses. Dans l'article on passe en revue quelaues découvertes faites pendant ces derniers ans à propos de l'influence de l'intéraction entre les éleves sur les résultats de lapprentissage. On met l'accent sur le résultat des recherches concernant la comparaison de differentes formes d'organisation sociale - cooperation, competition, individualisme- des activités scolaires, et sur quelques types d'interaction dont les effess positifs sur l'apprentissage s'averent particulierement remarquables. Ces donnies sont iues a laide de deux cadres theoriques différents, rbypotbese du conflit sociocognitif et lbypotbese de la regulation. Enfin, on énonce quelgues perspectives d"asenir pour quelgues unes des nombreuses questions qui restent encore sans réponse.

\section{Referencias}

DEL'TSCH, N.: “A theory of cooperation and competition". Human Relations, 1949, 2, $129^{-1}$ s.

DoISE, W., y MLGNY, G.: "Recherches socio-génétiques sur la coordination d'actions interdépendantes». Rerue Suisse de Psychologie, 1979, 34, 160-174.

Doise, W', y MLG.Ni, G.: "Factores sociológicos y psicosociológicos del desarrollo cognitivo». Anuario de Psicologia, 1978, 18, $21-40$.

Dolse, W.; MLGNy, G., y Perret-Clermont, A. N.: "Social interaction and the development of cognitive operations". European Journal of social Psychology, 1975, 5, 367-383.

Dori.1:, IX : "Paradigms for research on teacher effectiveness". Retiew of Research in Education, 1977. 9, $163-198$.

Forili., F. A.: The role of collaboration in problem-solving in children. Tesis doctoral inedita. Harvard Universily, Marzo 1981 .

FORMAN, F. A.: Understanding the role of peer interation in development. The contributions of Piaget and $\mathrm{V}$ ygotski. Comunicacion al Twelfth Annual Symposium de la Jean Piaget Society, Philadelphia, Pennsyilvania. Junio 1982 .

ForMAN, F. A., y CAzDEx, C. B.: "Fxploring Vygotskian perspectives in education: the cognitive value of peer interaction". En J. V. WERTSCH (Ed.); Culture, communication and cognition: L'ygotskian perspectives. Nueva York: Cambridge University Press, en prensa ( 1982$), 46$ p.

HAYES, L.: "The use of group contingencies for behavioral control: a review». Psychological Bulletin, 1976, 83, $628-648$.

Inhei.der, B.; Sinclair, H., y Bovet, M.: Apprentissage et structures de la connaissance. Paris: Presses Universitaires de France, 1974 /Aprendizaje y estructuras del conocimiento. Madrid: Morata, 1975 ].

JoHNSAN, D. W': “Student-student interaction: the neglected variable in education". Educational Researcber, 198 I (a), 10, s-10.

JohNSON, D. W.: "Social psychology». Fn F. M. FARI.EY and M. J. GORDON (Eds.), Psychology and education. The state of the union. Berkeley: McCutchan, 1981 (b), 26,-297.

JOHNSON, D. W.; JOH.NSON, R:: (Eds.) "Social interdependence in the classroom: cooperation, compctition and individualism." Journal of Research and Development in Education, 1978, 12, numero completo.

JoHNSoN, D. IW., y JoHNSoN, R.: "Conflict in the classroom: controversy and learning". Review of Educational Research, 1979, 49, \$1-70.

WHNSWN, D. W.; MARLYMA, G.; JohNSON, R.; NeisoN, D., y SKon, I.: “Fiffects of cooperative, comperitive, and individualistic gool structures on achievement: a meta-analysisn. Psychological Bulletin, $1981,89,47-62$.

LEVINA, R. E.: “L. S. Vygotski's ideas about the planning function of speech in children". En J. V.

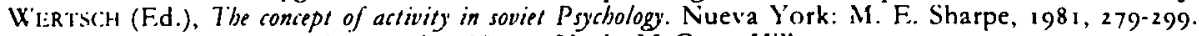

LEWIN, K.: A dynamic theory of personality. Nueva York: McGraw-Hill 1935.

MICHAEIS, J.: "Classroom reward structures and academic performance". Review of Educational Research, $1977,47,87-99$.

Pepitone, E. A. (Ed.): Children in cooperation and competition. Toward a developmental social psychology. Mass.: I.exington Books, 1980 .

PeRrti-CI.lRMont, A. N.: La construction de l'intelligence dans l'intéraction sociale. Berne: Petcr Lang, 1979 iLa construcción de la inteligencia en la interacioion social. Madrid: Visor/Aprendizaje, en prensa].

PERRET-CI.ERMONT, A. N.: “Perspectivas psicosociológicas del aprendizaje en situación colectiva». Infancia $y$ Aprendizaje, 1981, 16, 29-42.

Pingiar, J.:Le jugement moral chez lenfant. Paris: Alcan, 1932. (El criterio moral en el niño. Barcelona: Fontanella, 1971 ].

PingFt, J.: La psychologie de l'intelligence. Paris: Colin, 1947. [Psicologia de la inteligencia. Buenos Aires: Psiqué, 195.) 
Pliket, J.: L'équilibration des structures cognisives. Probleme central du développement. Paris: Presses Universitaires de France, 1975. (La equilibración de las estructuras operatorias. Madrid: Siglo XXI, 1978.]

Schubauer-Lfoni, M. L., y Perret-Ci.ermont, A. N.: “Intéractions sociales et représentations symboliques dans le cadre des problèmes auditifs". Recherches en Didactique des Mathématiques, 1980, 1, 297-350.

SI.AVIN, R.: «Classroom reward structure: analytical and practical reviewm. Review of Educational Research, $1980,50,241-272$.

VYGOTSKı, L. S.: «Aprendíizaje y desarrollo intelectual en la edad escolar». En LURIA, LeONTIEv, VYGotski y otros (compilación), Psicologia y Pedagogia. Madrid: A kal, 1973. [A rtículo escrito en 1934.]

WEBB, N. M.: "Student interaction and learning in small groups". Revies of Educational Research, 1982, J2, $421-44)$.

WEBB, N. M.: «Predicting learning from student interaction: defining the interaction variables». Educational Psychologist, $1983,18,33-4 \mathrm{I}$.

WERTSCH, J. V., y STONE, C. A.: "Microgenesis is a tool for developmental analysis». The Quarterly Newsletter of the Laboratory of comparative Human Cognition, 1978, 1, 8-10.

ZINCHENKo, V. P.: «Methodological problems in the psychological analysis of activity". En J. V. WERTSCH (Ed.), The concept of activity in soviet psychology. Nueva York: M. E. Sharpe, $198 \mathrm{I}$. 